MATHEMATICS OF COMPUTATION

Volume 76, Number 258, April 2007, Pages 1005-1023

S 0025-5718(06)01921-1

Article electronically published on October 30, 2006

\title{
HEURISTICS FOR CLASS NUMBERS AND LAMBDA INVARIANTS
}

\author{
JAMES S. KRAFT AND LAWRENCE C. WASHINGTON
}

\begin{abstract}
Let $K=\mathbf{Q}(\sqrt{-d})$ be an imaginary quadratic field and let $\mathbb{Q}(\sqrt{3 d})$ be the associated real quadratic field. Starting from the Cohen-Lenstra heuristics and Scholz's theorem, we make predictions for the behaviors of the 3-parts of the class groups of these two fields as $d$ varies. We deduce heuristic predictions for the behavior of the Iwasawa $\lambda$-invariant for the cyclotomic $\mathbf{Z}_{3}$ extension of $K$ and test them computationally.
\end{abstract}

The Cohen-Lenstra heuristics 1 give predictions for frequencies of class numbers and class groups of number fields. In the following, we investigate a related situation and a more specific question:

I. Are there heuristics for the Iwasawa lambda invariants, similar to those of Cohen and Lenstra for class groups of number fields? The $\lambda_{2}$-invariants of imaginary quadratic fields are given by a simple formula of Ferrero [4] and Kida [5] and are correspondingly not suitable for a heuristic analysis. We therefore consider the first nontrivial case, namely the $\lambda$-invariant for the cyclotomic $\mathbb{Z}_{3}$-extension of an imaginary quadratic field $K$ as $K$ varies. When 3 does not split in $K$, the frequency of $\lambda=0$ is easy to treat. We give a prediction for the frequency of $\lambda=1$ in this case. We also compute numerical data that agrees fairly well with the prediction; however, it is well known that the convergence of empirical data to the CohenLenstra heuristics is quite slow, and we presumably have a similar slowness in the present situation. Therefore, any numerical agreement or disagreement cannot necessarily be regarded as decisive. We also collect data for the case when 3 splits in the imaginary quadratic field. In this case, we always have $\lambda \geq 1$. It appears that the frequencies of a given $\lambda$ are similar to those for $\lambda-1$ in the nonsplit case. We regard this as pointing towards some type of theoretical model for $\lambda$ heuristics, similar to the idea of weighting by the inverse of the size of automorphism groups in the Cohen-Lenstra setting.

II. It was proved in 6 that if 3 splits in an imaginary quadratic field $\mathbb{Q}(\sqrt{-d})$ and if 3 divides the class number of $\mathbb{Q}(\sqrt{3 d})$, then $\lambda \geq 2$. Since it might be suspected that $\lambda$ tends to be small, this could indicate that 3 divides the class number of $\mathbb{Q}(\sqrt{3 d})$, with $d \equiv 2(\bmod 3)$, with less than the frequency predicted by CohenLenstra heuristics for class numbers of all real quadratic fields. Nevertheless, our numerical experiments do not indicate the presence of any such bias.

In Section 9 we give some data for the distribution of $\lambda_{3}$-invariants of imaginary quadratic fields $\mathbb{Q}(\sqrt{-d})$. As our analysis shows, it is natural to break into cases

Received by the editor August 23, 2005 and, in revised form, January 6, 2006.

2000 Mathematics Subject Classification. Primary 11R23, 11R29, 11R11.

(C)2006 American Mathematical Society 
according to the power of 3 dividing the class number $h^{+}$of the corresponding real quadratic field $\mathbb{Q}(\sqrt{3 d})$. After introducing some basic machinery in Section 1 and Section 2, we describe the Cohen-Lenstra heuristics in Section 3. The 3-part of the class group of an imaginary quadratic field $\mathbb{Q}(\sqrt{-d})$ is closely related to that of the associated real quadratic field $\mathbb{Q}(\sqrt{3 d})$ by Scholz's theorem, which says that their 3 -ranks differ by at most one. We need to consider the effect of this result in our study of $\lambda$. Therefore, in Section 4, we give several heuristic predictions related to Scholz's theorem. These are possibly of independent interest. In Section 5, we give a naive prediction for the probability that $\lambda=1$ and then, in Section 6, a more refined analysis. These two methods yield slightly different predictions, but they are close enough that it appears computationally impossible to differentiate between them. However, we give data for all of the steps that comprise the refined heuristics and therefore we believe that these give the correct prediction. In the last section, we briefly discuss the methods of calculating the data.

\section{BAsic NOTATiON}

Let $K$ be an imaginary quadratic field of discriminant $-d \neq-3$ with Dirichlet character $\chi$ and class number $h^{-}$. Let $L=K(\sqrt{-3})$ and $L^{+}=\mathbb{Q}(\sqrt{3 d})$. Then $L^{+}$ has discriminant

$$
D=3 d \quad \text { or } \quad d / 3 \text {. }
$$

Let $\epsilon, \chi^{+}$, and $h^{+}$be the fundamental unit, Dirichlet character, and class number of $L^{+}$, respectively. Let

$$
f(T)=f\left(T, \chi^{+}\right)=a_{0}+a_{1} T+a_{2} T^{2}+\cdots \in \mathbf{Z}_{3}[[T]]
$$

be the Iwasawa power series such that

$$
L_{3}\left(s, \chi^{+}\right)=f\left(4^{s}-1\right)
$$

is the 3 -adic $L$-function for $\chi^{+}$. Then

$$
f(0)=a_{0}=(1-\chi(3)) h^{-}, \quad f(3)=\left(1-\frac{\chi^{+}(3)}{3}\right) \frac{2 h^{+} \log _{3} \epsilon}{\sqrt{D}} .
$$

Let $\lambda$ be the Iwasawa $\lambda_{3}$ invariant for $K$. Then $\lambda$ is the smallest $n$ such that $a_{n} \not \equiv 0(\bmod 3)$. When $d \equiv 2(\bmod 3)$, so 3 splits in $K / \mathbb{Q}$, we have $\chi(3)=1$ and consequently $a_{0}=0$. In this case, therefore, we have $\lambda \geq 1$.

\section{UNITS AND CLASS NUMBERS}

Let $A^{+}$and $A^{-}$be the 3-Sylow subgroups of the ideal class groups of $L^{+}$and $K$. Let $r$ be the 3-rank of $A^{+}$and $s$ be the 3-rank of $A^{-}$. A theorem of Scholz [7] says that $r \leq s \leq r+1$.

Lemma 1. $\lambda \geq s$.

Proof. Let $K_{n}$ be the $n$-th layer of the cyclotomic $\mathbb{Z}_{3}$-extension of $K$. Since $K_{n} / K$ is totally ramified for all $n$, the norm map on the ideal class groups is surjective. Therefore, $A^{-}=A_{0}$ is a quotient of $X^{-}=\lim _{\leftarrow} A_{n}^{-}$. By [8, Corollary 13.29], $X^{-} \simeq \mathbb{Z}_{3}^{\lambda}$. The result follows easily. 
Dutarte [3] has predicted that the probability that $s=r+1$ is $3^{-(r+1)}$ and has shown that this is consistent with the Cohen-Lenstra heuristics. Since [3] is not widely available, and since we need the ideas of that paper, we briefly sketch the argument.

If $\mathfrak{p}$ is a prime ideal of $L$, let $U_{\mathfrak{p}}^{(j)}$ be the units of the completion of $L$ at $\mathfrak{p}$ that are congruent to $1 \bmod \mathfrak{p}^{j}$. Let $U^{(j)}=\prod U_{\mathfrak{p}}^{(j)}$, where the product is over the primes of $L$ above 3 . Then $u \in U^{(3)}$ if and only if $u \equiv 1(\bmod 3 \sqrt{-3})$ (that is, each component of $u$ satisfies this congruence). It can be shown that $U^{(1)} / U^{(3)}$ breaks into a direct sum of four eigenspaces for the action of $\operatorname{Gal}(L / \mathbf{Q})$, each of which is cyclic of order 3 . If $I$ is relatively prime to 3 and represents an ideal class of order 3 of $L$, then $I^{3}=(\alpha)$ and $\alpha^{8}$ (embedded diagonally) lies in $U^{(1)}$. Moreover, if $\alpha^{8} \in U^{(1)}$, then $L\left(\alpha^{1 / 3}\right) / L$ is unramified if and only if $\alpha^{8} \in U^{(3)}$. If $I$ comes from $K$ or $L^{+}$, then $\alpha^{8}$ lies in a corresponding eigenspace of $U^{(1)} / U^{(3)}$. Similarly, if $\epsilon$ is the fundamental unit of $L$, or of $L^{+}$, then $\epsilon^{8} \in U^{(1)}$, so we obtain an element of $U^{(1)} / U^{(3)}$ lying in the cyclic eigenspace corresponding to $L^{+}$. Dutarte assumed that this element is trivial with probability $1 / 3$. Moreover, if there are independent ideal classes $I_{1}, \ldots, I_{r}$ generating the elements of order 3 in $L^{+}$, then the probability that the corresponding $\alpha_{1}^{8}, \ldots, \alpha_{r}^{8}$ and $\epsilon^{8}$ generate the trivial subgroup of $U^{(1)} / U^{(3)}$ is $3^{-r-1}$. In Scholz's theorem, if the subgroup generated by these elements is trivial, then $s=r+1$. If the subgroup is nontrivial (hence is the full eigenspace of order 3 ), then $s=r$. Therefore, $s=r+1$ with probability $3^{-r-1}$.

Lemma 2. (i)

$$
\left(1-\frac{\chi^{+}(3)}{3}\right) \frac{2 \log _{3} \epsilon}{\sqrt{D}} \in \mathbb{Z}_{3}
$$

(ii) Assume 3 is not split in $K / \mathbb{Q}$ (equivalently, $d \not \equiv 2(\bmod 3))$. Let $\epsilon$ be the fundamental unit of $L^{+}$. Then $\left(1-\chi^{+}(3) / 3\right)\left(2 \log _{3} \epsilon\right) / \sqrt{D} \equiv 0(\bmod 3)$ if and only if $\epsilon^{8} \in U^{(3)}$.

Proof. (i) The expression lies in the completion of $L^{+}$at a prime above 3 . If this completion is not $\mathbb{Q}_{3}$, then the nontrivial element of the Galois groupof $L^{+} / \mathbb{Q}_{3}$ inverts $\epsilon$ (up to sign) and changes the sign of $\sqrt{D}$. Therefore, the expression is fixed by the Galois group, hence lies in $\mathbb{Q}_{3}$.

Since the residue fields for the primes above 3 have order 3 or 9 , we have $\epsilon^{2}$ or $\epsilon^{8}$ congruent to 1 mod the primes above 3 . For simplicity of exposition, we always work with $\epsilon^{8}$.

Suppose $d \not \equiv 0(\bmod 3)$. Let $\epsilon^{8}=1+\sqrt{3 d} \alpha$, with $\alpha \in \mathbb{Z}_{3}[\sqrt{3 d}]$. Then

$$
\log _{3}\left(\epsilon^{8}\right) \equiv \sqrt{3 d} \alpha-\frac{1}{2}(3 d) \alpha^{2}+\frac{1}{3}(3 d) \sqrt{3 d} \alpha^{3} \equiv 0 \quad(\bmod \sqrt{3 d})
$$

which yields the result, since $\chi^{+}(3)=0$. Now, suppose $d \equiv 0(\bmod 3)$. Then $\epsilon^{8} \equiv 1(\bmod 3)$, so $\log _{3}\left(\epsilon^{8}\right) \equiv 0(\bmod 3)$, which again yields the result.

(ii) Suppose $\epsilon^{8} \equiv 1(\bmod 3 \sqrt{-3})$. Then $\log _{3} \epsilon \equiv 0(\bmod 3 \sqrt{-3})$, as in (i). If $d \equiv 1(\bmod 3)$, then $\chi^{+}(3)=0$, so the result follows immediately. If $d \equiv 0$ $(\bmod 3)$, then $3 \nmid D$, but $\left(1-\chi^{+}(3) / 3\right)$ contributes 3 to the denominator. We find that

$$
\left(1-\frac{\chi^{+}(3)}{3}\right) \frac{2 \log _{3} \epsilon}{\sqrt{D}} \equiv 0 \quad(\bmod \sqrt{-3}) .
$$

But the left side is in $\mathbb{Z}_{3}$, so it must be congruent to $0 \bmod 3$. 
Conversely, suppose $\left(1-\chi^{+}(3) / 3\right)\left(2 \log _{3} \epsilon\right) / \sqrt{D} \equiv 0(\bmod 3)$. If $d \equiv 1(\bmod 3)$, then $\log _{3} \epsilon \equiv 0(\bmod 3 \sqrt{-3})$, and if $d \equiv 0(\bmod 3)$, then $\log _{3} \epsilon \equiv 0(\bmod 9)$. In both cases, $\eta=\exp \left(8 \log _{3} \epsilon\right)$ converges, with $\eta \equiv 1(\bmod 3 \sqrt{-3})$. Moreover, $\epsilon^{8}=\zeta \eta$ for some root of unity $\zeta$ because $\log _{3} \epsilon^{8}=\log _{3} \eta$. Since $\zeta=\epsilon^{8} / \eta \equiv 1$ $(\bmod \sqrt{-3})$, it follows that $\zeta$ is a 3 -power root of unity, which must be trivial or a cube root of unity since it is in the completion of $L$. But $\epsilon^{8}$ and $\eta$ lie in the completion of $L^{+}$. Since this completion does not contain a nontrivial cube root of unity, $\zeta=1$. It follows that $\epsilon^{8} \equiv 1(\bmod 3 \sqrt{-3})$, as desired.

Remark. Calculations indicate that part (ii) of the lemma is also true when $d \equiv 2$ $(\bmod 3)$, but we do not have a proof of this.

The main results that we need for our computations are contained in the following.

Theorem 1. Let $\epsilon^{8}=x+y \sqrt{D}$, with $2 x, 2 y \in \mathbb{Z}$, and let $\epsilon=x_{1}+y_{1} \sqrt{D}$ with $2 x_{1}, 2 y_{1} \in \mathbb{Z}$.

(i) Suppose $d \equiv 0(\bmod 3)$, so $3 \nmid D$. Then $\epsilon^{8} \in U^{(3)}$ if and only if $y \equiv 0$ $(\bmod 9)$.

(ii) Suppose $d \equiv 0(\bmod 3)$. Then $\lambda=0$ if and only if $3 \nmid h^{-}$. If $3 \mid h^{-}$, then $\lambda>1$ if and only if

$$
2 \chi^{+}(3) h^{+} \frac{y}{3} \equiv h^{-} \quad(\bmod 9) .
$$

If this congruence does not hold, then $\lambda=1$.

(iii) Suppose $d \not \equiv 0(\bmod 3)$. Then $\epsilon^{8} \in U^{(3)}$ if and only if $y \equiv 0(\bmod 3)$, which happens if and only if $y_{1} \equiv 0(\bmod 3)$.

(iv) Suppose that $d \equiv 1(\bmod 3)$ and assume that $3 \nmid h^{+}$. We have $\lambda=0$ if and only if $3 \nmid h^{-}$. If $3 \mid h^{-}$, then $\lambda>1$ if and only if $h^{+} y+h^{-} \equiv 0(\bmod 9)$. If $9 \mid h^{-}$, then $\lambda>1$ if and only if $y_{1} \equiv 0(\bmod 9)$.

(v) Suppose $d \equiv 1(\bmod 3)$ and assume that $3 \mid h^{+}$. Then $\lambda>1$ if and only if

$$
h^{+} y \equiv h^{-} \quad(\bmod 9) .
$$

If this congruence does not hold, then $\lambda=1$.

Proof. (i) Write $\epsilon^{8}=1+3 \alpha$ with $\alpha$ integral in $L^{+}$and $3 \alpha=x-1+y \sqrt{D}$. Since $x^{2}-D y^{2}=1$ and $x \equiv 1(\bmod 3)$, we must have $y \equiv 0(\bmod 3)$, hence $x \equiv 1$ $(\bmod 9)$. Therefore,

$$
\alpha=\frac{x-1}{3}+\frac{y}{3} \sqrt{D} \equiv \frac{y}{3} \sqrt{D} \quad(\bmod 3) .
$$

It follows that $\epsilon^{8} \in U^{(3)}$ if and only if $y \equiv 0(\bmod 9)$. This proves (i).

(ii) Since $a_{0}=h^{-}$when $d \equiv 0(\bmod 3)$, we have $\lambda=0 \Leftrightarrow 3 \nmid h^{-}$. Now assume $3 \mid h^{-}$. First, suppose $3 \nmid h^{+}$. Then $s=1>r=0$, so $\epsilon^{8} \in U^{(3)}$. By (i), $y \equiv 0$ $(\bmod 9)$. Since $x^{2}-D y^{2}=1$ and $x \equiv 1(\bmod 3)$, we have $x \equiv 1(\bmod 81)$. Therefore, $\epsilon^{8} \equiv 1+y \sqrt{D}(\bmod 81)$. It follows that

$$
\log _{3} \epsilon=\frac{1}{8} \log _{3} \epsilon^{8} \equiv \frac{1}{8} y \sqrt{D} \quad(\bmod 81)
$$

Therefore,

$$
h^{-}+3 a_{1}=a_{0}+3 a_{1} \equiv\left(1-\frac{\chi^{+}(3)}{3}\right) \frac{2 h^{+} \log _{3} \epsilon}{\sqrt{D}} \equiv \frac{3-\chi^{+}(3)}{3} \frac{1}{4} h^{+} y \quad(\bmod 9) .
$$


Since $9 \mid y$, the right-hand side of this congruence becomes $2 \chi^{+}(3)\left(h^{+}\right)(y / 3)(\bmod 9)$. Therefore, $\lambda>1 \Leftrightarrow 3 \mid a_{1} \Leftrightarrow 2 \chi^{+}(3)\left(h^{+}\right)(y / 3) \equiv h^{-}(\bmod 9)$.

Now suppose that $3 \mid h^{+}$. In this case, we have $\log _{3} \epsilon \equiv y \sqrt{D} / 8(\bmod 9)$. Since $\left(1-\chi^{+}(3) / 3\right) h^{+}$is integral,

$$
\left(1-\frac{\chi^{+}(3)}{3}\right) \frac{2 h^{+} \log _{3} \epsilon}{\sqrt{D}} \equiv\left(1-\frac{\chi^{+}(3)}{3}\right) 2 h^{+} \frac{y}{8} \equiv 2 \chi^{+}(3) h^{+} \frac{y}{3} \quad(\bmod 9) .
$$

Since $h^{-}=a_{0}$, it follows that

$$
h^{-}+3 a_{1} \equiv 2 \chi^{+}(3) h^{+} \frac{y}{3} \quad(\bmod 9) .
$$

Therefore, $h^{-}$is congruent to the right-hand side of this congruence if and only if $3 \mid a_{1}$, which happens if and only if $\lambda>1$. This completes the proof of (ii).

(iii) Write $\epsilon^{8}=1+(x-1)+y \sqrt{3 d}$, with $x \equiv 1(\bmod 3)$. If $\epsilon^{8} \in U^{(3)}$, then $y \equiv 0(\bmod 3)$. Conversely, suppose $y \equiv 0(\bmod 3)$. Since $x^{2}-3 d y^{2}=1$, we have $x \equiv 1(\bmod 27)$, so $\epsilon^{8} \in U^{(3)}$. The binomial theorem applied to $x_{1}+y_{1} \sqrt{3 d}$ yields $y \equiv 8 x_{1}^{7} y_{1}(\bmod 3)$. Since $x_{1}^{2}-3 d y_{1}^{2}= \pm 1$, we must have $x_{1} \not \equiv 0(\bmod 3)$. Therefore, $y \equiv 0(\bmod 3)$ if and only if $y_{1} \equiv 0(\bmod 3)$.

(iv) Since $a_{0}=2 h^{-}$when $d \equiv 1(\bmod 3)$, we have $\lambda=0 \Leftrightarrow 3 \nmid h^{-}$. Now suppose $3 \mid h^{-}$. Then $s=1>r=0$, so $\epsilon^{8} \in U^{(3)}$. By (iii), $y \equiv y_{1} \equiv 0(\bmod 3)$. Since $x^{2}-3 d y^{2}=1$ and $x \equiv 1(\bmod 3)$, we have $x \equiv 1(\bmod 27)$. Therefore, $\epsilon^{8} \equiv 1+y \sqrt{3 d}(\bmod 27)$. It follows that

$$
\log _{3} \epsilon=\frac{1}{8} \log _{3} \epsilon^{8} \equiv \frac{1}{8} y \sqrt{3 d} \quad(\bmod 27)
$$

Therefore,

$$
2 h^{-}+3 a_{1}=a_{0}+3 a_{1} \equiv \frac{2 h^{+} \log _{3} \epsilon}{\sqrt{3 d}} \equiv \frac{2 h^{+} y}{8} \equiv-2 h^{+} y \quad(\bmod 9) .
$$

Consequently,

$$
\lambda>1 \Leftrightarrow 3 \mid a_{1} \Leftrightarrow h^{+} y+h^{-} \equiv 0 \quad(\bmod 9)
$$

If $9 \mid h^{-}$, this becomes $\lambda>1 \Leftrightarrow 9 \mid y$. The binomial expansion of $\left(x_{1}+y_{1} \sqrt{3 d}\right)^{8}$ yields $y \equiv y_{1}\left(-x_{1}^{2}+6 y_{1}^{2}\right)(\bmod 9)$. As in (iii), $x_{1} \not \equiv 0(\bmod 3)$, so we find $y \equiv 0$ $(\bmod 9) \Leftrightarrow y_{1} \equiv 0(\bmod 9)$. This completes the proof of (iv).

(v) We have $\epsilon^{8}=1+(x-1+y \sqrt{3 d}) \equiv 1+y \sqrt{3 d}(\bmod 3)$, so

$$
\log _{3} \epsilon \equiv \frac{1}{8}\left(y \sqrt{3 d}+\frac{1}{3}(y \sqrt{3 d})^{3}\right) \equiv-\left(y+y^{3} d\right) \sqrt{3 d} \equiv y \sqrt{3 d} \quad(\bmod 3),
$$

since $d \equiv 1(\bmod 3)$. Therefore, since $3 \mid h^{+}$and $\chi^{+}(3)=0$,

$$
\left(1-\frac{\chi^{+}(3)}{3}\right) \frac{2 h^{+} \log _{3} \epsilon}{\sqrt{3 d}} \equiv 2 h^{+} y \quad(\bmod 3 \sqrt{-3}),
$$

hence mod 9 , since both sides are in $\mathbb{Z}_{3}$. In the present case, $a_{0}=(1-\chi(3)) h^{-}=$ $2 h^{-}$, so

$$
2 h^{-}+3 a_{1} \equiv 2 h^{+} y \quad(\bmod 9) .
$$

The results of part (v) follow easily.

Note that, when $d \equiv 2(\bmod 3)$ and $3 \mid h^{+}$, if we try to obtain information on $\lambda$ as in (ii) and (v), we obtain $0 \equiv 0$ since $\log _{3} \epsilon \equiv 0(\bmod 3)$ and $a_{0}=0$. However, $\lambda>1$, so the analogue of (ii) and (v) is trivially true. 
For part (ii), computing $\epsilon$, raising to the eighth power to obtain $\epsilon^{8}$, and then extracting $y$ causes a significant slowdown in the computations for large discriminants. However, this can be avoided by computing $\epsilon$ and then raising it to the 8th power mod 27 .

\section{Cohen-Lenstra heuristics}

The Cohen-Lenstra heuristics predict the following, where

$$
\eta=\prod_{i=1}^{\infty}\left(1-3^{i}\right) \approx .5601 .
$$

Let $G$ be a finite abelian 3-group. The probability that the 3-Sylow subgroup of the ideal class group of $K$ is $G$ is

$$
\operatorname{Prob}\left(A^{-} \simeq G\right)=\frac{\eta}{\# \operatorname{Aut}(G)} .
$$

The probability that the 3-Sylow subgroup of the ideal class group of $L^{+}$is $G$ is

$$
\operatorname{Prob}\left(A^{+} \simeq G\right)=\frac{3 \eta}{2 \# G \# \operatorname{Aut}(G)} .
$$

Throughout the following, we assume that these heuristic predictions are correct. This yields the following corollaries:

$$
\begin{gathered}
\operatorname{Prob}\left(3 \| h^{-}\right)=\eta / 2 \approx .2801, \\
\operatorname{Prob}\left(3 \| h^{+}\right)=\eta / 4 \approx .1400, \\
\operatorname{Prob}\left(9 \mid h^{+}\right)=1-\frac{7}{4} \eta \approx .0198 . \\
\operatorname{Prob}\left(A^{+} \text {is cyclic } \geq 9\right)=\eta / 32 \approx .0175
\end{gathered}
$$

(we write "cyclic $\geq 9$ " for "cyclic of order $\geq 9$ ").

The first and second of these follow immediately by letting $G=\mathbf{Z} / 3 \mathbf{Z}$. The third follows by evaluating $1-\operatorname{Prob}\left(3 \nmid h^{+}\right)-\operatorname{Prob}\left(3 \| h^{+}\right)$. The fourth is obtained by summing the appropriate geometric series.

Throughout the paper, we make the assumption that these heuristic predictions hold when $d$ is restricted to a fixed congruence class mod 3 . Numerical calculations support this hypothesis.

\section{Predictions for Class groups}

Throughout this paper, we make the assumption that the only constraint that $A^{+}$puts on $A^{-}$is via Scholz's theorem. Namely, the rank of one group affects the rank of the other, but the exponent of one group does not affect the other. In particular, if we know that $A^{-}$has rank 1 , then the distribution of possible orders of $A^{-}$is the same whether or not we assume in addition that 9 divides $h^{+}$.

For example, we assume the equality of the conditional probabilities

$$
\begin{gathered}
\operatorname{Prob}\left(3 \| h^{-} \mid A^{+} \text {cyclic } \geq 3\right)=\operatorname{Prob}\left(3 \| h^{-} \mid A^{+} \text {cyclic } \geq 9\right) \\
=\operatorname{Prob}\left(3\left\|h^{-} \mid 3\right\| h^{+}\right) .
\end{gathered}
$$

Note that any two of these equalities imply the third. We will give data supporting these assumptions.

We need to find values for these conditional probabilities. 
Prediction 1. (i) $\operatorname{Prob}\left(3\left\|h^{-} \mid 3\right\| h^{+}\right)=\frac{16}{27} \approx .5926$.

(ii) $\operatorname{Prob}\left(3 \| h^{-} \mid A^{+}\right.$cyclic $\left.\geq 9\right)=\frac{16}{27}$.

(iii) $\operatorname{Prob}\left(3 \| h^{-} \mid A^{+}\right.$cyclic $\left.\geq 3\right)=\frac{16}{27}$.

Derivations. We are assuming that all three are equal, so we give the reasoning that yields (ii). The others are essentially the same. Since

$$
\operatorname{Prob}\left(3 \| h^{-}, A^{+} \text {cyclic } \geq 9\right)=\operatorname{Prob}\left(3 \| h^{-}, A^{+} \text {cyclic } \geq 9, r=s=1\right),
$$

it follows that

$$
\begin{gathered}
\operatorname{Prob}\left(3 \| h^{-} \mid A^{+} \text {cyclic } \geq 9\right) \\
=\operatorname{Prob}\left(3 \| h^{-} \mid A^{+} \text {cyclic } \geq 9, s=r=1\right) \cdot \operatorname{Prob}\left(s=r=1 \mid A^{+} \text {cyclic } \geq 9\right) .
\end{gathered}
$$

We make two assumptions:

First, note that if $s=1$, then by definition $A^{-}$is cyclic. We regard $A^{-}$as a random cyclic group of order $3^{n}$ with $n \geq 1$ and calculate the probability that it is cyclic of order 3 :

$$
\frac{\operatorname{Prob}\left(A^{-} \text {cyclic } 3\right)}{\operatorname{Prob}\left(A^{-} \text {cyclic } \geq 3\right)}=\frac{\eta / \phi(3)}{\sum_{n \geq 1} \eta / \phi\left(3^{n}\right)}=\frac{2}{3} .
$$

This might be a dangerous assumption since we are assuming that a higher power of 3 (namely, 9) dividing $h^{+}$has no effect. But, as discussed above, our hypothesis is that the only effect that $A^{+}$has on $A^{-}$is via the ranks, not the orders, as in Scholz's theorem.

Second, as mentioned above, Dutarte has predicted that

$$
\operatorname{Prob}\left(s=r=1 \quad \mid A^{+} \text {is cyclic } \geq 3\right)=\frac{8}{9} .
$$

We again assume that the rank is what is important here, and therefore that

$$
\operatorname{Prob}\left(s=r=1 \quad A^{+} \text {is cyclic } \geq 9\right)=\frac{8}{9} .
$$

Putting these two assumptions together, we obtain

$$
\operatorname{Prob}\left(3 \| h^{-} \mid A^{+} \text {is cyclic } \geq 9\right)=\frac{2}{3} \times \frac{8}{9}=\frac{16}{27} .
$$

This is prediction (ii).

We can test these predictions directly, but we prefer to transform them since we need the other forms later.

\section{Prediction 2.}

$$
\operatorname{Prob}\left(3 \| h^{-}|9| h^{+}\right)=\frac{\eta / 54}{1-7 \eta / 4} \approx .5244 .
$$

Derivation. Note that if $A^{+}$is noncyclic, then $A^{-}$has rank at least 2 , so $9 \mid h^{-}$. Therefore,

$$
\begin{gathered}
\operatorname{Prob}\left(3 \| h^{-}, 9 \mid h^{+}\right)=\operatorname{Prob}\left(3 \| h^{-}, A^{+} \text {cyclic } \geq 9\right) \\
=\operatorname{Prob}\left(3 \| h^{-} \mid A^{+} \text {cyclic } \geq 9\right) \cdot \operatorname{Prob}\left(A^{+} \text {cyclic } \geq 9\right) \\
=\frac{16}{27} \times \frac{\eta}{32}=\frac{\eta}{54} .
\end{gathered}
$$

Dividing by $\operatorname{Prob}\left(9 \mid h^{+}\right)=1-7 \eta / 4$ yields the result. 
TABLE 1. $\operatorname{Prob}\left(3 \| h^{-}|9| h^{+}\right)$

\begin{tabular}{|c|c|c|c|}
\hline $10^{9}<d<10^{9}+10^{7}$ & $9 \mid h^{+}$ & $9 \mid h^{+}$and $3 \| h^{-}$ & Ratio \\
\hline$d \equiv 0(\bmod 3)$ & 13627 & 7435 & .5456 \\
$d \equiv 1(\bmod 3)$ & 20941 & 11408 & .5448 \\
$d \equiv 2(\bmod 3)$ & 20970 & 11345 & .5410 \\
\hline \hline $10^{14}<d<10^{14}+10^{7}$ & & & \\
\hline$d \equiv 0(\bmod 3)$ & 15065 & 7900 & .5244 \\
$d \equiv 1(\bmod 3)$ & 22506 & 11796 & .5241 \\
$d \equiv 2(\bmod 3)$ & 22382 & 11789 & .5267 \\
\hline
\end{tabular}

Note that Prediction 1(ii) and Prediction 2 are equivalent to each other under the assumption of the Cohen-Lenstra heuristics. Both predictions can be checked numerically, and we give data for Prediction 2 in Table 1.

The present form of Prediction 2 requires evaluating the class number $h^{+}$for each $d$ and the calculations proceed relatively slowly. It is possible to speed up the empirical testing by using a Bayesian trick:

$$
\begin{gathered}
\operatorname{Prob}\left(3 \| h^{-}|9| h^{+}\right) \\
=\operatorname{Prob}\left(9\left|h^{+}\right| 3 \| h^{-}\right) \frac{\operatorname{Prob}\left(3 \| h^{-}\right)}{\operatorname{Prob}\left(9 \mid h^{+}\right)} \\
=\operatorname{Prob}\left(9\left|h^{+}\right| 3 \| h^{-}\right) \frac{\eta / 2}{1-7 \eta / 4} .
\end{gathered}
$$

Therefore, the Cohen-Lenstra heuristics imply that Prediction 2 is equivalent to the following.

Prediction 3. $\operatorname{Prob}\left(9\left|h^{+}\right| 3 \| h^{-}\right)=\frac{1}{27} \approx .0370$.

TABLE 2. $\operatorname{Prob}\left(9\left|h^{+}\right| 3 \| h^{-}\right)$

\begin{tabular}{|c|c|c|c|}
\hline $10^{9}<d<10^{9}+10^{7}$ & $3 \| h^{-}$ & $3 \| h^{-}$and $9 \mid h^{+}$ & Ratio \\
\hline$d \equiv 0(\bmod 3)$ & 209501 & 7435 & .0355 \\
$d \equiv 1(\bmod 3)$ & 313102 & 11408 & .0364 \\
$d \equiv 2(\bmod 3)$ & 314069 & 11345 & .0361 \\
\hline \hline $10^{14}<d<10^{14}+10^{7}$ & & & \\
\hline$d \equiv 0(\bmod 3)$ & 212523 & 7900 & .0372 \\
$d \equiv 1(\bmod 3)$ & 318745 & 11796 & .0370 \\
$d \equiv 2(\bmod 3)$ & 318815 & 11789 & .0370 \\
\hline
\end{tabular}

Table 2 indicates that the prediction of $1 / 27$ is reasonable. The calculations to test this prediction are faster since we evaluate $h^{-}$for each $d$, which is faster than evaluating $h^{+}$. However, the situation is slightly more subtle than it might appear at first. If $h^{-}$is evaluated first, then $\eta / 2 \approx 0.2801$ of the values of $d$ require an evaluation of $h^{+}$. When $h^{+}$is evaluated first, only $\eta / 32 \approx .0198$ of the values of $d$ require $h^{+}$to be evaluated. But the evaluation of $h^{+}$takes enough longer that evaluating $h^{-}$first is still faster. 
The prediction that

$$
\operatorname{Prob}\left(3\left\|h^{-} \mid 3\right\| h^{+}\right)=\frac{16}{27}
$$

can be tested similarly, as follows. We have

$$
\begin{gathered}
\operatorname{Prob}\left(3\left\|h^{-} \mid 3\right\| h^{+}\right)=\operatorname{Prob}\left(3\left\|h^{+} \mid 3\right\| h^{-}\right) \frac{\operatorname{Prob}\left(3 \| h^{-}\right)}{\operatorname{Prob}\left(3 \| h^{+}\right)} \\
=2 \operatorname{Prob}\left(3\left\|h^{+} \mid 3\right\| h^{-}\right),
\end{gathered}
$$

which yields the following.

Prediction 4. $\operatorname{Prob}\left(3\left\|h^{+} \mid 3\right\| h^{-}\right)=\frac{8}{27} \approx .2963$.

Table 3 gives data for this situation.

TABle $3 . \operatorname{Prob}\left(3\left\|h^{+} \mid 3\right\| h^{-}\right)$

\begin{tabular}{|c|c|c|c|}
\hline $10^{9}<d<10^{9}+10^{7}$ & $3 \| h^{-}$ & $3 \| h^{-}$and $3 \| h^{+}$ & Ratio \\
\hline$d \equiv 0(\bmod 3)$ & 209501 & 60052 & .2866 \\
$d \equiv 1(\bmod 3)$ & 313102 & 90361 & .2886 \\
$d \equiv 2(\bmod 3)$ & 314069 & 90754 & .2890 \\
\hline \hline $10^{14}<d<10^{14}+10^{7}$ & & & \\
\hline$d \equiv 0(\bmod 3)$ & 212523 & 62583 & .2945 \\
$d \equiv 1(\bmod 3)$ & 318745 & 94001 & .2949 \\
$d \equiv 2(\bmod 3)$ & 318815 & 93991 & .2948 \\
\hline
\end{tabular}

The data in Tables 1, 2 and 3 agree with Predictions 2 and 3 and Prediction 4, which are equivalent to Prediction 1(ii) and Prediction 1(i), respectively. If these two are correct, then it follows easily that Prediction 1(iii) is also correct.

We also need to consider the probability that $3 \| h^{-}$given that $3 \| h^{+}$, but broken into finer subsets. Recall that if $\epsilon$ is the fundamental unit of $L^{+}$, then $\epsilon^{8} \in U^{(1)}$. A method for determining whether $\epsilon^{8} \in U^{(3)}$ is given in Theorem 1 .

Prediction 5. (i) $\operatorname{Prob}\left(3\left\|h^{-}, 3\right\| h^{+}, \epsilon^{8} \in U^{(3)}\right)=\eta / 27 \approx .0207$.

(ii) $\operatorname{Prob}\left(3\left\|h^{-}, 3\right\| h^{+}, \epsilon^{8} \notin U^{(3)}\right)=\eta / 9 \approx .0622$.

Derivations. (i)

$$
\begin{gathered}
\operatorname{Prob}\left(3\left\|h^{-}, 3\right\| h^{+}, \epsilon^{8} \in U^{(3)}\right) \\
=\operatorname{Prob}\left(3\left\|h^{-}, 3\right\| h^{+}, \epsilon^{8} \in U^{(3)}, s=r=1\right) \\
=\operatorname{Prob}\left(3\left\|h^{-} \mid 3\right\| h^{+}, \epsilon^{8} \in U^{(3)}, s=r=1\right) \\
\times \operatorname{Prob}\left(s=r=1 \mid 3 \| h^{+}, \epsilon^{8} \in U^{(3)}\right) \\
\times \operatorname{Prob}\left(\epsilon^{8} \in U^{(3)} \mid 3 \| h^{+}\right) \times \operatorname{Prob}\left(3 \| h^{+}\right) .
\end{gathered}
$$

Dutarte's analysis shows that we should expect $\epsilon^{8} \in U^{(3)}$ to happen with probability $1 / 3$, independent of conditions on $h^{+}$. Therefore, we assume that

$$
\operatorname{Prob}\left(\epsilon^{8} \in U^{(3)} \mid 3 \| h^{+}\right)=\frac{1}{3} .
$$


Dutarte's analysis also indicates that we should have

$$
\operatorname{Prob}\left(s=r=1 \mid 3 \| h^{+}, \epsilon^{8} \in U^{(3)}\right)=\frac{2}{3} .
$$

This is deduced as follows. If $3 \| h^{+}$, then $r=1$. We are assuming $\epsilon^{8}$ is trivial in $U^{(1)} / U^{(3)}$. The underlying idea in Dutarte's analysis is that if $\alpha$ comes from a nontrivial ideal class of order 3 in $L^{+}$, then the location of $\alpha^{8}$ in the group $U^{(1)} / U^{(3)}$ is independent of $\epsilon$ and therefore is trivial with probability $1 / 3$. The probability that $\alpha$ is nontrivial is therefore $2 / 3$. Consequently, $\epsilon^{8}$ and $\alpha$ generate the full eigenspace, hence $s=r$, with probability $2 / 3$.

TABle 4. $\operatorname{Prob}\left(3\left\|h^{-}, 3\right\| h^{+}, \epsilon^{8} \notin U^{(3)}\right)$

\begin{tabular}{|c|c|c|c|}
\hline $10^{9}<d<10^{9}+10^{6}$ & Total & $3\left\|h^{-}, 3\right\| h^{+}, \epsilon^{8} \notin U^{(3)}$ & Ratio \\
\hline$d \equiv 0(\bmod 3)$ & 75999 & 4508 & .0593 \\
$d \equiv 1(\bmod 3)$ & 113999 & 6790 & .0596 \\
$d \equiv 2(\bmod 3)$ & 113964 & 6750 & .0592 \\
\hline \hline $10^{11}<d<10^{11}+10^{6}$ & & & \\
\hline$d \equiv 0(\bmod 3)$ & 76012 & 4708 & .0619 \\
$d \equiv 1(\bmod 3)$ & 113986 & 6781 & .0595 \\
$d \equiv 2(\bmod 3)$ & 113967 & 6940 & .0609 \\
\hline
\end{tabular}

If $s=1$, then $A^{-}$is cyclic. We assume that the conditions $3 \| h^{+}$and $\epsilon^{8} \in U^{(3)}$ have no additional effect and that therefore $A^{-}$is a random nontrivial cyclic 3group, so

$$
\operatorname{Prob}\left(3\left\|h^{-} \mid 3\right\| h^{+}, \epsilon^{8} \in U^{(3)}, s=r=1\right)=\frac{2}{3},
$$

as in the derivation of Prediction 1.

Putting everything together, we obtain

$$
\operatorname{Prob}\left(3\left\|h^{-}, 3\right\| h^{+}, \epsilon^{8} \in U^{(3)}\right)=\frac{2}{3} \times \frac{2}{3} \times \frac{1}{3} \times \frac{\eta}{4}=\frac{\eta}{27} .
$$

We now derive (ii). The probabilities in (i) and (ii) add to give

$\operatorname{Prob}\left(3\left\|h^{-}, 3\right\| h^{+}\right)=\operatorname{Prob}\left(3\left\|h^{-} \mid 3\right\| h^{+}\right) \cdot \operatorname{Prob}\left(3 \| h^{+}\right)=\frac{16}{27} \times \frac{\eta}{4}=\frac{4 \eta}{27}$.

Therefore, (ii) follows from (i).

\section{NAIVE HeURISTICS FOR $\lambda$ IN THE NONSPlit CASE}

In this section and the next, we consider the case where 3 does not split in $K / \mathbb{Q}$. The constant term $a_{0}$ of the power series $f(T)$ is $(1-\chi(3)) h^{-}$. Since $\chi(3)=0$ or -1 , the constant term is divisible by 3 if and only if $3 \mid h^{-}$. Therefore, the Cohen-Lenstra heuristics yield the following.

Prediction 6. If 3 does not split in $K$, then $\operatorname{Prob}(\lambda=0)=\eta \approx .5601$.

It follows that $3 \mid a_{0}$, hence $\lambda \geq 1$, with probability $1-\eta$. If we assume that each of the remaining coefficients is divisible by 3 with probability $1 / 3$, then we have

$$
\operatorname{Prob}(\lambda=j)=(1-\eta)\left(\frac{1}{3}\right)^{j-1}\left(\frac{2}{3}\right) .
$$


In particular,

$$
\operatorname{Prob}(\lambda=1)=\frac{2}{3}(1-\eta) \approx 0.2932 .
$$

In the following, we give a more refined analysis that predicts that this probability should be $\eta / 2 \approx 0.2801$, which is computationally indistinguishable from 0.2932 .

\section{REFINED HEURISTICS FOR $\lambda$ IN THE NONSPLIT CASE}

In the present section, we again consider the case

$$
d \not \equiv 2 \quad(\bmod 3) .
$$

In other words, we assume that 3 does not split in $K / \mathbb{Q}$. Our goal is to estimate the probability that $\lambda=1$. We divide into cases corresponding to the power of 3 dividing $h^{+}$and show how the ideas behind Cohen-Lenstra heuristics and Dutarte's analysis can be combined to give an estimate for $\operatorname{Prob}(\lambda=1)$ that is slightly different than the naive heuristic given in the previous section.

We also assume that the heuristics derived in Section 4 hold when $d$ runs through a fixed residue class mod 3. This assumption agrees with the numerical data.

6.1. Case I: $9 \mid h^{+}$.

Lemma 3. Assume that $d \not \equiv 2(\bmod 3)$ and $9 \mid h^{+}$. Then $\lambda=1 \Longleftrightarrow 3 \| h^{-}$.

Proof. Since $9 \mid h^{+}$, Lemma2 2 implies that $a_{0}+3 a_{1} \equiv f(3) \equiv 0(\bmod 9)$. Therefore, $3\left|a_{1} \Longleftrightarrow 9\right| a_{0}$. Since $a_{0}=(1-\chi(3)) h^{-}$and $\chi(3) \neq 1$, the lemma follows.

From Prediction 2 and Lemma 3 ,

$$
\operatorname{Prob}\left(\lambda=1|9| h^{+}\right)=\operatorname{Prob}\left(3 \| h^{-}|9| h^{+}\right)=\frac{\eta / 54}{1-7 \eta / 4} \approx 0.5244 .
$$

Table 1 contains data for this prediction (because of Lemma 3).

Also, multiplying by $\operatorname{Prob}\left(9 \mid h^{+}\right)=1-7 \eta / 4$ yields

$$
\operatorname{Prob}\left(\lambda=1,9 \mid h^{+}\right)=\operatorname{Prob}\left(3 \| h^{-}, 9 \mid h^{+}\right)=\frac{\eta}{54} \approx .0104 .
$$

This is somewhat higher than the entries .0067, .0079, .0076, .0103 in Tables 13, 14 , [15] and 16. Note, however, that the total fraction of fields with $9 \mid h^{+}$is also smaller than the predicted $1-7 \eta / 4 \approx .0198$. This is an example of the slowness of convergence to the Cohen-Lenstra predictions. Larger discriminants yield the values in Table 5, which are close to the prediction.

TABle 5. $\operatorname{Prob}\left(\lambda=1,9 \mid h^{+}\right)$

\begin{tabular}{|c|c|c|c|}
\hline $10^{9}<d<10^{9}+10^{7}$ & Total & $\lambda=1$ and $9 \mid h^{+}$ & Ratio \\
\hline$d \equiv 0(\bmod 3)$ & 759907 & 7435 & .0098 \\
$d \equiv 1(\bmod 3)$ & 1139873 & 11408 & .0100 \\
\hline \hline $10^{11}<d<10^{11}+10^{7}$ & & & \\
\hline$d \equiv 0(\bmod 3)$ & 759937 & 7621 & .0100 \\
$d \equiv 1(\bmod 3)$ & 1139886 & 11632 & .0102 \\
\hline
\end{tabular}


6.2. Case II: $3 \nmid h^{+}$. Since $\lambda=0 \Leftrightarrow 3 \nmid h^{-}$, and $3 \nmid h^{-} \Rightarrow 3 \nmid h^{+}$,

$$
\begin{gathered}
\operatorname{Prob}\left(\lambda=0 \mid 3 \nmid h^{+}\right)=\operatorname{Prob}\left(3 \nmid h^{+} \mid \lambda=0\right) \frac{\operatorname{Prob}(\lambda=0)}{\operatorname{Prob}\left(3 \nmid h^{+}\right)} \\
=1 \cdot \frac{\operatorname{Prob}\left(3 \nmid h^{-}\right)}{3 \eta / 2}=\frac{2}{3} .
\end{gathered}
$$

This means that if $3 \nmid h^{+}$, then 3 divides $a_{0}$ with probability $1 / 3$. It seems reasonable to assume that in this case, for each $n \geq 1$, we also have that 3 divides $a_{n}$ with probability $1 / 3$. If this is the case, then

$$
\operatorname{Prob}\left(\lambda=1 \mid 3 \nmid h^{+}\right)=\frac{1}{3} \times \frac{2}{3}=\frac{2}{9} \approx .2222 .
$$

Table 6 contains numerical data for this situation.

TABle 6. $\operatorname{Prob}\left(\lambda=1 \mid 3 \nmid h^{+}\right)$

\begin{tabular}{|c|c|c|c|}
\hline $10^{9}<d<10^{9}+10^{6}$ & $3 \nmid h^{+}$ & $\lambda=1$ & Ratio \\
\hline$d \equiv 0(\bmod 3)$ & 64676 & 14345 & .2218 \\
$d \equiv 1(\bmod 3)$ & 96669 & 21104 & .2183 \\
\hline \hline $10^{11}<d<10^{11}+10^{6}$ & & & \\
\hline$d \equiv 0(\bmod 3)$ & 64113 & 14331 & .2235 \\
$d \equiv 1(\bmod 3)$ & 96365 & 21299 & .2210 \\
\hline
\end{tabular}

Multiplying by $\operatorname{Prob}\left(3 \nmid h^{+}\right)$yields

$$
\operatorname{Prob}\left(\lambda=1,3 \nmid h^{+}\right)=\frac{2}{9} \times \frac{3 \eta}{2}=\frac{\eta}{3} \approx .1867 .
$$

This agrees well with the entries in Tables 9, 10, 11, and 12,

We remark that $\lambda=0$ if and only if $f(3) \equiv f(0) \not \equiv 0(\bmod 3)$, which happens if and only if $\epsilon^{8} \notin U^{(3)}$, by Lemma 2. The above prediction of $2 / 3$ for $\lambda=0$ agrees with the prediction given by Dutarte for $\epsilon^{8} \notin U^{(3)}$.

6.3. Case III: $3 \| h^{+}$. We have

$$
a_{0}+3 a_{1} \equiv\left(1-\frac{\chi^{+}(3)}{3}\right) \frac{2 h^{+} \log _{3} \epsilon}{\sqrt{D}} \quad(\bmod 9) .
$$

By Lemma 2, $\left(1-\chi^{+}(3) / 3\right) 2 \log _{3}(\epsilon) / \sqrt{D} \in \mathbb{Z}_{3}$. If $3 \| h^{+}$, then we must have $3 \mid a_{0}$, so $\lambda \geq 1$ (this also follows from Scholz's theorem). We need to consider two cases, depending on $\epsilon$.

(1) If $\epsilon^{8} \in U^{(3)}$, then Theorem 1 implies that $\lambda=1 \Leftrightarrow 3 \| h^{-}$. It follows that

$$
\operatorname{Prob}\left(\lambda=1,3 \| h^{+}, \epsilon^{8} \in U^{(3)}\right)=\operatorname{Prob}\left(3\left\|h^{-}, 3\right\| h^{+}, \epsilon^{8} \in U^{(3)}\right)=\frac{\eta}{27},
$$

by Prediction 5 .

(2) Now suppose $\epsilon^{8} \notin U^{(3)}$. If $9 \mid h^{-}$and $3 \| h^{+}$, then Theorem 1 implies that $\lambda=1$. We therefore have

$$
\begin{gathered}
\operatorname{Prob}\left(\lambda=1,9 \mid h^{-}, 3 \| h^{+}, \epsilon^{8} \notin U^{(3)}\right) \\
=\operatorname{Prob}\left(9 \mid h^{-}, 3 \| h^{+}, \epsilon^{8} \notin U^{(3)}\right) \\
=\operatorname{Prob}\left(3 \| h^{+}, \epsilon^{8} \notin U^{(3)}\right)-\operatorname{Prob}\left(3\left\|h^{-}, 3\right\| h^{+}, \epsilon^{8} \notin U^{(3)}\right) .
\end{gathered}
$$


One of Dutarte's basic assumptions is that $3 \| h^{+}$and $\epsilon^{8} \notin U^{(3)}$ are independent events. Therefore, we obtain (using Prediction 5)

$$
\begin{gathered}
\operatorname{Prob}\left(3 \| h^{+}\right) \cdot \operatorname{Prob}\left(\epsilon^{8} \notin U^{(3)}\right)-\frac{\eta}{9} \\
=\frac{\eta}{4} \times \frac{2}{3}-\frac{\eta}{9}=\frac{\eta}{18} .
\end{gathered}
$$

If $3 \| h^{-}$and $3 \| h^{+}$, then parts (ii) and (v) of Theorem 1 say that $\lambda>1$ if and only if a congruence mod 9 holds. Since $\epsilon \notin U^{(3)}$, both sides of this congruence are divisible by 3 but not by 9 , so each side is either 3 or $6 \bmod 9$. We assume that the two sides are congruent with probability $1 / 2$. In Table 7 , we give data to support this assumption.

TABLE $7 . \lambda$ when $3\left\|h^{+}, 3\right\| h^{-}, \epsilon^{8} \notin U^{(3)}$

\begin{tabular}{|c|c|c|c|}
\hline $10^{9}<d<10^{9}+10^{6}$ & $\lambda=1$ & $\lambda \geq 2$ & Ratio \\
\hline$d \equiv 0(\bmod 3)$ & 2308 & 2200 & 1.0491 \\
$d \equiv 1(\bmod 3)$ & 3352 & 3438 & .9750 \\
\hline \hline $10^{11}<d<10^{11}+10^{6}$ & & & \\
\hline$d \equiv 0(\bmod 3)$ & 2374 & 2334 & 1.0171 \\
$d \equiv 1(\bmod 3)$ & 3414 & 3367 & 1.0140 \\
\hline
\end{tabular}

Under the assumption that the cases $\lambda=1$ and $\lambda \geq 2$ are equally probable, we have

$$
\begin{aligned}
& \operatorname{Prob}\left(\lambda=1,3\left\|h^{-}, 3\right\| h^{+}, \epsilon^{8} \notin U^{(3)}\right) \\
= & \frac{1}{2} \operatorname{Prob}\left(3\left\|h^{-}, 3\right\| h^{+}, \epsilon^{8} \notin U^{(3)}\right)=\frac{\eta}{18} .
\end{aligned}
$$

It follows that

$$
\begin{gathered}
\operatorname{Prob}\left(\lambda=1,3 \| h^{+}, \epsilon^{8} \notin U^{(3)}\right) \\
=\operatorname{Prob}\left(\lambda=1,9 \mid h^{-}, 3 \| h^{+}, \epsilon^{8} \notin U^{(3)}\right)+\operatorname{Prob}\left(\lambda=1,3\left\|h^{-}, 3\right\| h^{+}, \epsilon^{8} \notin U^{(3)}\right) \\
=\frac{\eta}{18}+\frac{\eta}{18}=\frac{\eta}{9} .
\end{gathered}
$$

Putting together cases 1 and 2, we obtain

$$
\operatorname{Prob}\left(\lambda=1,3 \| h^{+}\right)=\frac{\eta}{27}+\frac{\eta}{9}=\frac{4 \eta}{27} \approx .0830
$$

This can also be expressed as

$$
\operatorname{Prob}\left(\lambda=1 \mid 3 \| h^{+}\right)=\frac{16}{27} \approx .5926
$$

Table 8 contains data for this situation. 
TABLe 8. $\operatorname{Prob}\left(\lambda=1 \mid 3 \| h^{+}\right)$

\begin{tabular}{|c|c|c|c|}
\hline $10^{9}<d<10^{9}+10^{6}$ & $3 \| h^{+}$ & $\lambda=1$ & Ratio \\
\hline$d \equiv 0(\bmod 3)$ & 9967 & 6000 & .6020 \\
$d \equiv 1(\bmod 3)$ & 15185 & 8979 & .5908 \\
\hline \hline $10^{11}<d<10^{11}+10^{6}$ & & & \\
\hline$d \equiv 0(\bmod 3)$ & 10432 & 6210 & .5953 \\
$d \equiv 1(\bmod 3)$ & 15486 & 9241 & .5967 \\
\hline
\end{tabular}

\section{The PRobability THAT $\lambda=1$}

Adding up the cases where $9 \mid h^{+}, 3 \nmid h^{+}$, and $3 \| h^{+}$, we obtain

\section{Prediction 7.}

$$
\begin{aligned}
\operatorname{Prob}(\lambda=1) & =\frac{\eta}{54}+\frac{\eta}{3}+\frac{4 \eta}{27}=\frac{\eta}{2} \\
& \approx .0104+.1867+.0830=.2801 .
\end{aligned}
$$

Is this prediction correct? Or is the naive prediction correct? In Tables 9 through 12, we give data that agrees well with the refined prediction. However, in most situations, the convergence to the Cohen-Lenstra heuristics is notoriously slow, so this is not conclusive. The naive heuristics give .2932, and the refined heuristic is the sum of three terms. Suppose that exactly one of the four numbers $.2932, .0104, .1867, .0830$ is incorrect. If the .0104 is incorrect, then it would have to be replaced by .0235 , which is larger than $\operatorname{Prob}\left(9 \mid h^{+}\right)=1-7 \eta / 4 \approx .0198$. This is impossible if we believe the Cohen-Lenstra heuristics. If the .0830 is wrong, then it should be .0961 , which is possible, but unlikely since .0830 is closer to the data. The most questionable assumption made in the calculation of .0830 was that two quantities agree or do not agree mod 9 with equal probabilities. This is the same type of reasoning that is used in the naive heuristics, and in the present case the assumption is supported fairly well by the data. If the probability of $\lambda=1$ was .7119 instead of .5 , then the present heuristics would yield the same result as the naive reasoning. But a probability this far from .5 is not supported by the data. Finally, note that the .1867 was obtained by naive reasoning in the same spirit as used to obtain the .2932 , so it is very unlikely that .1867 is wrong and .2932 is right. Moreover, the "naive" assumption used to obtain the .1867 seems more easy to justify than the overall naive assumption, since in the case $3 \nmid h^{+}$we already had $3 \mid a_{0}$ with probability $1 / 3$, while the overall naive assumption was made simply on the basis of not knowing anything better. Therefore, the best guess seems to be that the naive heuristics are incorrect.

\section{The SPlit CASE}

The distribution of lambda invariants for the split case $(d \equiv 2(\bmod 3))$ is very similar to that in the other cases, except that the lambda values are one larger. This is presumably caused by the fact that $a_{0}=0$, so the Iwasawa power series is $T$ times a power series: $f(T)=T g(T)$. Moreover, Ferrero and Greenberg have shown that $g(0) \neq 0$, which is similar to the nonsplit case where $f(0) \neq 0$. This 
seems to indicate that there should be a heuristic model for lambda invariants in terms of modules

$$
\mathbb{Z}_{3}[[T]] /(g(T))
$$

where $g(0) \neq 0$. We have not yet found such a model.

As mentioned in the introduction, one of our motivations for this study was to see if the frequency of $3 \nmid h^{+}$is higher than expected when $d \equiv 2(\bmod 3)$. The Cohen-Lenstra prediction is $3 \eta / 2 \approx .8402$. For $10^{6}<d<10^{6}+2 \times 10^{5}$ we have .8682 and for $10^{7}<d<10^{7}+2 \times 10^{5}$ we have .8618. These agree fairly well, especially since smaller values of $d$ are usually less likely to have $3 \mid h^{+}$, so the frequency of $3 \nmid h^{+}$tends to be higher than predicted. Also, the results for $d \equiv 2$ $(\bmod 3)$ are very similar to those for $d \not \equiv 2(\bmod 3)$. Therefore, we conclude that the $\lambda$-invariant does not cause a bias in $h^{+}$.

\section{DATA ON $\lambda$ INVARIANTS}

In Tables 9 through 12, we gave data on lambda invariants. By restricting to $\lambda=0,1$ and $\lambda \geq 2$, we were able to use Theorem 1 to perform the computations for much larger discriminants, since the algorithms to compute class numbers and fundamental units are subexponential. In contrast, the methods used to compute larger values of lambda (see the next section) are of the order of magnitude of the discriminant. In Tables 13 through 18, we give the results of our computations that include larger values of lambda, broken into the three congruence classes of $d$ mod 3. We also give the fraction of the total for each entry.

TABLE $9.10^{9}<d<10^{9}+10^{6}, \quad d \equiv 0(\bmod 3)$

\begin{tabular}{|c|c|c|c|c|}
\hline$d \equiv 0(\bmod 3)$ & $\lambda=0$ & $\lambda=1$ & $\lambda \geq 2$ & Total \\
\hline $3 \nmid h^{+}$ & 43326 & 14345 & 7005 & 64676 \\
$3 \| h^{+}$ & 0 & 6000 & 3967 & 9967 \\
$9 \mid h^{+}$ & 0 & 750 & 606 & 1356 \\
\hline Totals & 43326 & 21095 & 11578 & 75999 \\
\hline $3 \nmid h^{+}$ & .5701 & .1888 & .0922 & .8510 \\
$3 \| h^{+}$ & 0 & .0789 & .0522 & .1311 \\
$9 \mid h^{+}$ & 0 & .0099 & .0080 & .0178 \\
\hline Totals & .5701 & .2776 & .1523 & 1.000 \\
\hline
\end{tabular}

TABLE $10.10^{11}<d<10^{11}+10^{6}, \quad d \equiv 0(\bmod 3)$

\begin{tabular}{|c|c|c|c|c|}
\hline$d \equiv 0(\bmod 3)$ & $\lambda=0$ & $\lambda=1$ & $\lambda \geq 2$ & Total \\
\hline $3 \nmid h^{+}$ & 42868 & 14331 & 6914 & 64113 \\
$3 \| h^{+}$ & 0 & 6210 & 4222 & 10432 \\
$9 \mid h^{+}$ & 0 & 741 & 726 & 1467 \\
\hline Totals & 42868 & 21282 & 11862 & 76012 \\
\hline $3 \nmid h^{+}$ & .5640 & .1885 & .0910 & .8435 \\
$3 \| h^{+}$ & 0 & .0817 & .0555 & .1372 \\
$9 \mid h^{+}$ & 0 & .0097 & .0096 & .0193 \\
\hline Totals & .5640 & .2800 & .1561 & 1.000 \\
\hline
\end{tabular}


TABLE $11.10^{9}<d<10^{9}+10^{6}, \quad d \equiv 1(\bmod 3)$

\begin{tabular}{|c|c|c|c|c|}
\hline$d \equiv 1(\bmod 3)$ & $\lambda=0$ & $\lambda=1$ & $\lambda \geq 2$ & Total \\
\hline $3 \nmid h^{+}$ & 65155 & 21104 & 10410 & 96669 \\
$3 \| h^{+}$ & 0 & 8971 & 6214 & 15185 \\
$9 \mid h^{+}$ & 0 & 1203 & 942 & 2145 \\
\hline Totals & 65155 & 31278 & 17566 & 113999 \\
\hline $3 \nmid h^{+}$ & .5715 & .1851 & .0913 & .8480 \\
$3 \| h^{+}$ & 0 & .0787 & .0545 & .1332 \\
$9 \mid h^{+}$ & 0 & .0106 & .0083 & .0188 \\
\hline Totals & .5715 & .2744 & .1541 & 1.000 \\
\hline
\end{tabular}

TABLE $12.10^{11}<d<10^{11}+10^{6}, \quad d \equiv 1(\bmod 3)$

\begin{tabular}{|c|c|c|c|c|}
\hline$d \equiv 1(\bmod 3)$ & $\lambda=0$ & $\lambda=1$ & $\lambda \geq 2$ & Total \\
\hline $3 \nmid h^{+}$ & 64547 & 21299 & 10519 & 96365 \\
$3 \| h^{+}$ & 0 & 9241 & 6245 & 15486 \\
$9 \mid h^{+}$ & 0 & 1164 & 971 & 2135 \\
\hline Totals & 64547 & 31704 & 17735 & 113986 \\
\hline $3 \nmid h^{+}$ & .5663 & .1869 & .0923 & .8454 \\
$3 \| h^{+}$ & 0 & .0811 & .0548 & .1359 \\
$9 \mid h^{+}$ & 0 & .0102 & .0085 & .0187 \\
\hline Totals & .5663 & .2781 & .1556 & 1.000 \\
\hline
\end{tabular}

TABLE $13.10^{6}<d<10^{6}+2 \cdot 10^{5}, \quad d \equiv 0(\bmod 3)$

\begin{tabular}{|c|c|c|c|c|c|c|}
\hline$d \equiv 0(\bmod 3)$ & $\lambda=0$ & $\lambda=1$ & $\lambda=2$ & $\lambda=3$ & $\lambda \geq 4$ & Total \\
\hline $3 \nmid h^{+}$ & 8979 & 2820 & 971 & 290 & 159 & 13219 \\
$3 \| h^{+}$ & 0 & 1162 & 432 & 157 & 60 & 1811 \\
$9 \mid h^{+}$ & 0 & 102 & 46 & 16 & 8 & 172 \\
\hline Totals & 8979 & 4084 & 1449 & 463 & 227 & 15202 \\
\hline $3 \nmid h^{+}$ & .5906 & .1855 & .0639 & .0191 & .0105 & .8696 \\
$3 \| h^{+}$ & 0 & .0764 & .0284 & .0103 & .0039 & .1191 \\
$9 \mid h^{+}$ & 0 & .0067 & .0030 & .0011 & .0005 & .0113 \\
\hline Totals & .5906 & .2686 & .0953 & .0305 & .0149 & 1.000 \\
\hline
\end{tabular}

TABLe $14.10^{7}<d<10^{7}+2 \cdot 10^{5}, \quad d \equiv 0(\bmod 3)$

\begin{tabular}{|c|c|c|c|c|c|c|}
\hline$d \equiv 0(\bmod 3)$ & $\lambda=0$ & $\lambda=1$ & $\lambda=2$ & $\lambda=3$ & $\lambda \geq 4$ & Total \\
\hline $3 \nmid h^{+}$ & 8838 & 2823 & 922 & 318 & 180 & 13081 \\
$3 \| h^{+}$ & 0 & 1132 & 474 & 181 & 101 & 1888 \\
$9 \mid h^{+}$ & 0 & 120 & 67 & 29 & 7 & 223 \\
\hline Totals & 8838 & 4075 & 1463 & 528 & 288 & 15192 \\
\hline $3 \nmid h^{+}$ & .5818 & .1858 & .0607 & .0209 & .0118 & .8610 \\
$3 \| h^{+}$ & 0 & .0745 & .0312 & .0119 & .0066 & .1243 \\
$9 \mid h^{+}$ & 0 & .0079 & .0044 & .0019 & .0005 & .0147 \\
\hline Totals & .5818 & .2682 & .0963 & .0348 & .0190 & 1.000 \\
\hline
\end{tabular}


TABLE $15.10^{6}<d<10^{6}+2 \cdot 10^{5}, \quad d \equiv 1(\bmod 3)$

\begin{tabular}{|c|c|c|c|c|c|c|}
\hline$d \equiv 1(\bmod 3)$ & $\lambda=0$ & $\lambda=1$ & $\lambda=2$ & $\lambda=3$ & $\lambda \geq 4$ & Total \\
\hline $3 \nmid h^{+}$ & 13399 & 4271 & 1364 & 439 & 260 & 19733 \\
$3 \| h^{+}$ & 0 & 1706 & 695 & 241 & 115 & 2757 \\
$9 \mid h^{+}$ & 0 & 174 & 78 & 35 & 20 & 307 \\
\hline Totals & 13399 & 6151 & 2137 & 715 & 395 & 22797 \\
\hline $3 \nmid h^{+}$ & .5878 & .1873 & .0598 & .0193 & .0114 & .8656 \\
$3 \| h^{+}$ & 0 & .0748 & .0305 & .0106 & .0050 & .1209 \\
$9 \mid h^{+}$ & 0 & .0076 & .0034 & .0015 & .0009 & .0135 \\
\hline Totals & .5878 & .2698 & .0937 & .0314 & .0173 & 1.0000 \\
\hline
\end{tabular}

TABle $16 \cdot 10^{7}<d<10^{7}+2 \cdot 10^{5}, \quad d \equiv 1(\bmod 3)$

\begin{tabular}{|c|c|c|c|c|c|c|}
\hline$d \equiv 1(\bmod 3)$ & $\lambda=0$ & $\lambda=1$ & $\lambda=2$ & $\lambda=3$ & $\lambda \geq 4$ & Total \\
\hline $3 \nmid h^{+}$ & 13175 & 4244 & 1409 & 468 & 220 & 19516 \\
$3 \| h^{+}$ & 0 & 1769 & 745 & 258 & 129 & 2901 \\
$9 \mid h^{+}$ & 0 & 234 & 95 & 41 & 14 & 384 \\
\hline Totals & 13175 & 6247 & 2249 & 767 & 363 & 22801 \\
\hline $3 \nmid h^{+}$ & .5778 & .1861 & .0618 & .0205 & .0096 & .8559 \\
$3 \| h^{+}$ & 0 & .0776 & .0327 & .0113 & .0057 & .1272 \\
$9 \mid h^{+}$ & 0 & .0103 & .0042 & .0018 & .0006 & .0168 \\
\hline Totals & .5778 & .2740 & .0986 & .0336 & .0159 & 1.0000 \\
\hline
\end{tabular}

TABLE $17.10^{6}<d<10^{6}+2 \cdot 10^{5}, \quad d \equiv 2(\bmod 3)$

\begin{tabular}{|c|c|c|c|c|c|c|}
\hline$d \equiv 2(\bmod 3)$ & $\lambda=0$ & $\lambda=1$ & $\lambda=2$ & $\lambda=3$ & $\lambda \geq 4$ & Total \\
\hline $3 \nmid h^{+}$ & 0 & 13418 & 4242 & 1410 & 717 & 19787 \\
$3 \| h^{+}$ & 0 & 0 & 1650 & 672 & 361 & 2683 \\
$9 \mid h^{+}$ & 0 & 0 & 186 & 84 & 51 & 321 \\
\hline Totals & 0 & 13418 & 6078 & 2166 & 1129 & 22791 \\
\hline $3 \nmid h^{+}$ & 0 & .5887 & .1861 & .0619 & .0315 & .8682 \\
$3 \| h^{+}$ & 0 & 0 & .0724 & .0295 & .0158 & .1177 \\
$9 \mid h^{+}$ & 0 & 0 & .0082 & .0037 & .0022 & .0141 \\
\hline Totals & 0 & .5887 & .2667 & .0950 & .0495 & 1.0000 \\
\hline
\end{tabular}

TABLE $18.10^{7}<d<10^{7}+2 \cdot 10^{5}, \quad d \equiv 2(\bmod 3)$

\begin{tabular}{|c|c|c|c|c|c|c|}
\hline$d \equiv 2(\bmod 3)$ & $\lambda=0$ & $\lambda=1$ & $\lambda=2$ & $\lambda=3$ & $\lambda \geq 4$ & Total \\
\hline $3 \nmid h^{+}$ & 0 & 13351 & 4189 & 1385 & 724 & 19649 \\
$3 \| h^{+}$ & 0 & 0 & 1713 & 696 & 380 & 2789 \\
$9 \mid h^{+}$ & 0 & 0 & 198 & 104 & 59 & 361 \\
\hline Totals & 0 & 13351 & 6100 & 2185 & 1163 & 22799 \\
\hline $3 \nmid h^{+}$ & 0 & .5856 & .1837 & .0607 & .0318 & .8618 \\
$3 \| h^{+}$ & 0 & 0 & .0751 & .0305 & .0167 & .1223 \\
$9 \mid h^{+}$ & 0 & 0 & .0087 & .0046 & .0026 & .0158 \\
\hline Totals & 0 & .5856 & .2676 & .0958 & .0510 & 1.0000 \\
\hline
\end{tabular}




\section{Calculating Lambda}

To calculate the lambda invariants, we first calculated $h^{+}$. If $h^{+}$was not divisible by 3 , we computed $h^{-}$. If $h^{-}$was not divisible by 3 , then $\lambda=0$ when $d \not \equiv 2(\bmod 3)$. In the other cases $\lambda \geq 1$, and we used the formulas from [2]. In the cases considered in the present paper, the formulas are as follows. Let $d_{0}=d$ if $3 \nmid d$ and $d_{0}=d / 3$ if $3 \mid d$, and let $A=\sum_{j=1}^{d_{0}-1} j \chi(4+9 j)$ and $B=\sum_{j=1}^{d_{0}-1} j \chi(7+9 j)$.

I. If $A \not \equiv B(\bmod 3)$, then $\lambda=1$.

II. If $A \equiv B \not \equiv 0(\bmod 3)$, then $\lambda=2$. If $A \equiv B \equiv 0(\bmod 3)$, then $\lambda \geq 3$.

III. If $\lambda \geq 3$, let $3 \leq m<9$ be the least integer (if it exists) such that

$$
\sum_{l=1}^{8} \sum_{k=0}^{2} A_{k, l} \sum_{j=1}^{d_{0}-1} j \chi(l+9 k+27 j) \not \equiv 0 \quad(\bmod 3),
$$

where

$$
A_{k, l}=\left(\begin{array}{c}
-\left(4\left(9-l^{2}\right) / 3\right) l^{2}\left(1-l^{2}+9 k l\right)+\left(1-l^{2}\right)^{3} / 9 \\
m
\end{array}\right) .
$$

Then $\lambda=m$.

Note that the computation becomes much longer whenever $\lambda \geq 3$. However, a considerable speedup is obtained by evaluating the binomial coefficients explicitly in advance and using only those terms for which the binomial coefficients are nonzero $\bmod 3$.

Note that the formula in [2] is missing the term $\left(1-l^{2}\right)^{3} / 9$ in the binomial coefficient. This corresponds to the fact that a term $(j p)^{3} / 3$ is missing in the calculation of $\log _{p}(i)$ in [2, page 104]. This term is needed only when $p=3$. However, it appears that the correct formula was used in the computations in that paper, since their values of $\lambda$ agree with those we computed with the corrected formula.

The computations were done with PARI. Most of the computations were done by both authors with separate programs on separate machines, and the results agreed.

\section{REFERENCES}

[1] H. Cohen and H. W. Lenstra, Jr., "Heuristics on class groups of number fields," Number Theory, Noordwijkerhout, 1983, Springer Lecture Notes in Math. 1068 (1984), 33-62. MR 0756082 (85j:11144)

[2] D. Dummit, D. Ford, H. Kisilevsky, and J. Sands, "Computation of Iwasawa lambda invariants for imaginary quadratic fields," J. Number Theory, 37 (1991), 100-121. MR1089792 (92a:11124)

[3] P. Dutarte, "Compatabilité avec le Spiegelungssatz de probabilités conjecturales sur le prang du groupe des classes," Théorie des Nombres, Publ. Math. de la Faculté des Sciences de Besançon, Année 1983-1984, 11pp. MR0803700 (86m:11103)

[4] B. Ferrero, "The cyclotomic $\mathbf{Z}_{\mathbf{2}}$-extension of imaginary quadratic fields," Amer. J. Math., 102 (1980), 447-459. MR0573095 (81g:12006)

[5] Y. Kida, "On cyclotomic $\mathbf{Z}_{\mathbf{2}}$-extensions of imaginary quadratic fields," Tôhoku Math. J., 31 (1979), 91-96. MR0526512 (80d:12003)

[6] J. Kraft, "Class numbers and Iwasawa invariants of quadratic fields," Proc. Amer. Math. Soc., 124 (1996), 31-34. MR.1301510 (96d:11112)

[7] A. Scholz, "Über die Beziehung der Klassenzahlen quadratischen Körper zueinander," J. reine angew. Math., 166 (1931), 201-203.

[8] L. Washington, Introduction to Cyclotomic Fields, Springer-Verlag, 1987. MR0718674 (85g:11001) 
The Ingenuity Project, Baltimore Polytechnic Institute, 1400 W. Cold Spring Lane, Baltimore, Maryland 21209

E-mail address: jkraft31@comcast.net

Department of Mathematics, University of Maryland, College Park, Maryland 20742

E-mail address: lcw@math.umd.edu 